\title{
Shengxuening versus oral iron supplementation for the treatment of renal anemia: A systematic review
}

\author{
Qin Zeng ${ }^{1,2}$, Xin-Hui Wang', Li-Ping Yang ${ }^{3}$, Rui Lang ${ }^{2}$, \\ Ying Liang ${ }^{2}$, Ren-Huan $\mathbf{Y u}^{2}$ \\ ${ }^{1}$ Graduate School of Beijing University of Chinese Medicine, Beijing 100029, China; \\ 2Department of Nephrology, Xiyuan Hospital of China Academy of Chinese Medical Sciences, \\ Beijing 100029, China; \\ ${ }^{3}$ Department of Nephrology, Guanganmen Hospital of China Academy of Chinese Medical Sciences, \\ Beijing 100053, China
}

Address for Correspondence: Prof. Ren-Huan Yu, MD, PhD, Department of Nephrology, Xiyuan Hospital of China Academy of Chinese

Medical Sciences, Beijing 100029 ,

Medical sciences, Being 100029 ,

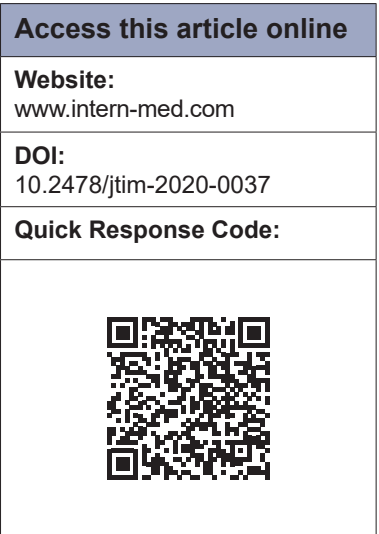

\section{ABSTRACT}

Shengxuening (SXN), as an effective supplement to heme-like iron, has been widely used in China to treat renal anemia. However, proof of its use for improving inflammation is scarce in the past decades. This work aimed to evaluate the effectiveness of SXN with inflammatory factors as primary endpoints. By searching PubMed, Embase, Cochrane Library, China National Knowledge Infrastructure (CNKI), China Biology Medicine disc (CBM), VIP Information/ China Science and Technology Journal Database, and WANFANG Database, we identified previous studies that met the inclusion criteria and included them in the systematic review. Analyses were performed using STATA. Nine randomized controlled trials were included in this systematic review. The results revealed that, when compared with oral iron supplementation, SXN can reduce the level of inflammatory factors, including hs-CRP (WMD $-1.93 \mathrm{mg} / \mathrm{L} ; 95 \%$ $\mathrm{Cl}-2.14$ to -1.72$)$, IL-6 $(P<0.05)$, and TNF- $\alpha(P<0.05)$, and significantly enhance the level of $\mathrm{Hb}$ (WMD $13.40 \mathrm{~g} / \mathrm{L} ; 95 \% \mathrm{Cl} 12.95$ to 13.84), TSAT (WMD 6.88\%; 95\% Cl 6.50 to 7.26 ), and SF (WMD $38.46 \mu \mathrm{g} / \mathrm{L} ; 95 \% \mathrm{Cl} 23.26$ to 53.67). Moreover, SXN exhibits a superior security than oral iron supplementation with less gastrointestinal adverse reactions (RR 0.14; 95\% $\mathrm{Cl} 0.06$ to 0.32 ). In patients with renal anemia, SXN is more effective and safer than oral iron supplementation, especially in reducing the level of inflammation.

Key words: Shengxuening, inflammation, renal anemia, oral iron supplementation, systematic review

\section{INTRODUCTION}

Anemia as a frequent complication of chronic kidney disease (CKD) is a risk factor of increasing mortality. ${ }^{[1]}$ As a matter of fact, common causes of renal anemia include relative erythropoietin (EPO) deficiency, disordered iron homeostasis, circulating uremic-induced inhibitors of erythropoiesis, shortened red blood cell survival, and various metabolic and mechanical factors. ${ }^{[2,3]}$ KDIGO (Kidney Disease: Improving Global Outcomes) recommended erythropoiesis-stimulating agents (ESAs) and iron supplementation as first-line treatments for patients with renal anemia in 2012. ${ }^{[4]}$ However, there still have been a lot of clinical cases in which sufficient supplementation of EPO and iron cannot correct anemia. The study has shown that, aside from EPO and iron deficiency, inflammation and hepcidin are also involved in the mechanisms of renal anemia, which have renewed interest in understanding the molecular mechanisms of anemia in CKD. ${ }^{[2]}$ A new therapy for effectively targeting the underlying pathophysiology of renal anemia is highly desirable but remains challenging.

Shengxuening (SXN), as an effective supplement to heme-like iron, has been widely used in China to treat renal anemia. 
A number of clinical studies have indicated that SXN tablets were highly effective for iron deficiency anemia, blood loss anemia, and perinatal anemia. ${ }^{[5]}$ Zhang $\mathrm{L}$ et al..$^{\left[{ }^{[6]}\right.}$ have done a systematic review, which showed that SXN is more effective and safer than ferrous succinate and ferrous sulfate in the treatment of renal anemia in 2016. On the one hand, iron supplementation is administered either orally or intravenously, while it is generally accepted that intravenous iron supplementation has a higher risk of increasing inflammatory reaction than oral iron supplementation. On the other hand, recently, new studies have shown that SXN could exhibit good improvement on inflammatory reaction in patients with renal anemia, while without systematic assessment on the effect as yet. Therefore, we decided to make a systematic review that evaluated the attribute of SXN versus oral iron supplementation, with inflammatory factors as primary endpoints, for the treatment of renal anemia.

\section{METHODS}

\section{Data sources and search strategy}

We searched PubMed, Embase, Cochrane Library, China National Knowledge Infrastructure (CNKI), China Biology Medicine disc (CBM), VIP Information/China Science and Technology Journal Database, and WANFANG Database for eligible articles from database inception to February 2020. We used the following combined title/abstract and Medical Subject Headings (MeSH terms: "Shengxuening" OR "Faeces bombycis extract" OR "Sodium iron chlorophyllin" AND "Renal Insufficiency, Chronic" OR "Renal dialysis" AND "Anemia" AND "Randomized controlled trials." The complete search used for PubMed was: (Shengxuening [Title/Abstract] OR Faeces bombycis extract [Title/Abstract] OR Sodium iron chlorophyllin [Title/Abstract]) AND (Renal Insufficiency, Chronic [Mesh] OR Kidney Insufficiency, Chronic [Title/Abstract] OR Chronic Kidney Diseases [Title/Abstract] OR Chronic Renal Diseases [Title/Abstract] OR Nephropathy [Title/ Abstract] OR Hemodialysis [Title/Abstract] OR Peritoneal dialysis [Title/Abstract] OR End-stage renal disease [Title/ Abstract]) AND (Renal anemia [Title/Abstract] OR Anemia [Title/Abstract]) AND (Randomized controlled trials [Title/Abstract] OR Random [Title/Abstract]). References of relevant articles were manually searched for additional potentially relevant trials.

\section{Study selection}

Trials satisfying the following inclusion criteria were included: (1) randomized controlled trial (RCT) design in both English and Chinese languages, (2) adult (age $\geq 18$ years) patients with renal anemia defined by KDIGO in 2012, (3) comparison of SXN and oral iron supplementation, and (4) primary endpoint was hypersensitive $\mathrm{C}$-reactive protein (hs-CRP). Interleukin-6 (IL-6), interleukin-8 (IL-8), tumor necrosis factor- $\alpha$ (TNF- $\alpha$ ), hemoglobin $(\mathrm{Hb})$, serum transferrin saturation (TSAT), serum ferritin (SF), and adverse reactions were secondary endpoints. Trials were excluded if: (1) different regimens applied between two treatment groups apart from SXN and oral iron supplementation; (2) kidney transplant patients; (3) patients with other chronic disease, which influences the inflammatory state; and (4) retrospective studies or duplicated publication. Two reviewers (Qin Zeng and Xin-Hui Wang) independently inspected the retrieved literatures, based on the inclusion criteria. For possibly relevant articles or in cases of disagreement between the two reviewers, we independently inspected the full article.

\section{Data extraction and quality assessment}

Two reviewers (Qin Zeng and Xin-Hui Wang) independently extracted data from included trials and assessed methods quality. In case of disagreement between the two reviewers, a third reviewer (Li-Ping Yang) extracted the data and guaranteed the results attained to reach a consensus. Data extracted included the first author's name, publication year, trial design, sample sizes, age, sex, baseline hs-CRP, Hb, TSAT, SF, doses of SXN and oral iron supplementation, duration of treatment, outcome measures, and adverse reactions.

We evaluated all potentially relevant sources of bias, including random sequence generation, allocation concealment, blinding of participants and personnel, binding of outcome assessment, incomplete outcome data, selective reporting, and other bias. We graded each domain as low risk of bias, unclear risk (lack of information or uncertainty over the potential for bias), or high risk of bias according to criteria specified in the Cochrane Handbook, version 5.1.0..$^{[7]}$

\section{Statistical analysis}

In this systematic review, pooled effect sizes were summarized as weighted mean difference (WMD) or standard mean difference (SMD) with its 95\% confidence intervals (CI) for continuous data. Dichotomous data were pooled as the risk ratio (RR) with 95\% CI. The chi-square test and Cochrane Q statistic were used for assessment of heterogeneity. Significant heterogeneity was defined as the $I^{2}$ value $\geq 50 \%$ and the Cochrane's Q statistic $P<$ 0.10. A random effect model was selected when significant heterogeneity was identified. Otherwise, a fixed-effect model was used.

In order to find the reason of significant heterogeneity, subgroup analysis was conducted for the primary endpoint and several secondary endpoints (Hb, TSAT, SF, adverse reactions) by patient type (non-dialysis versus dialysis). All 


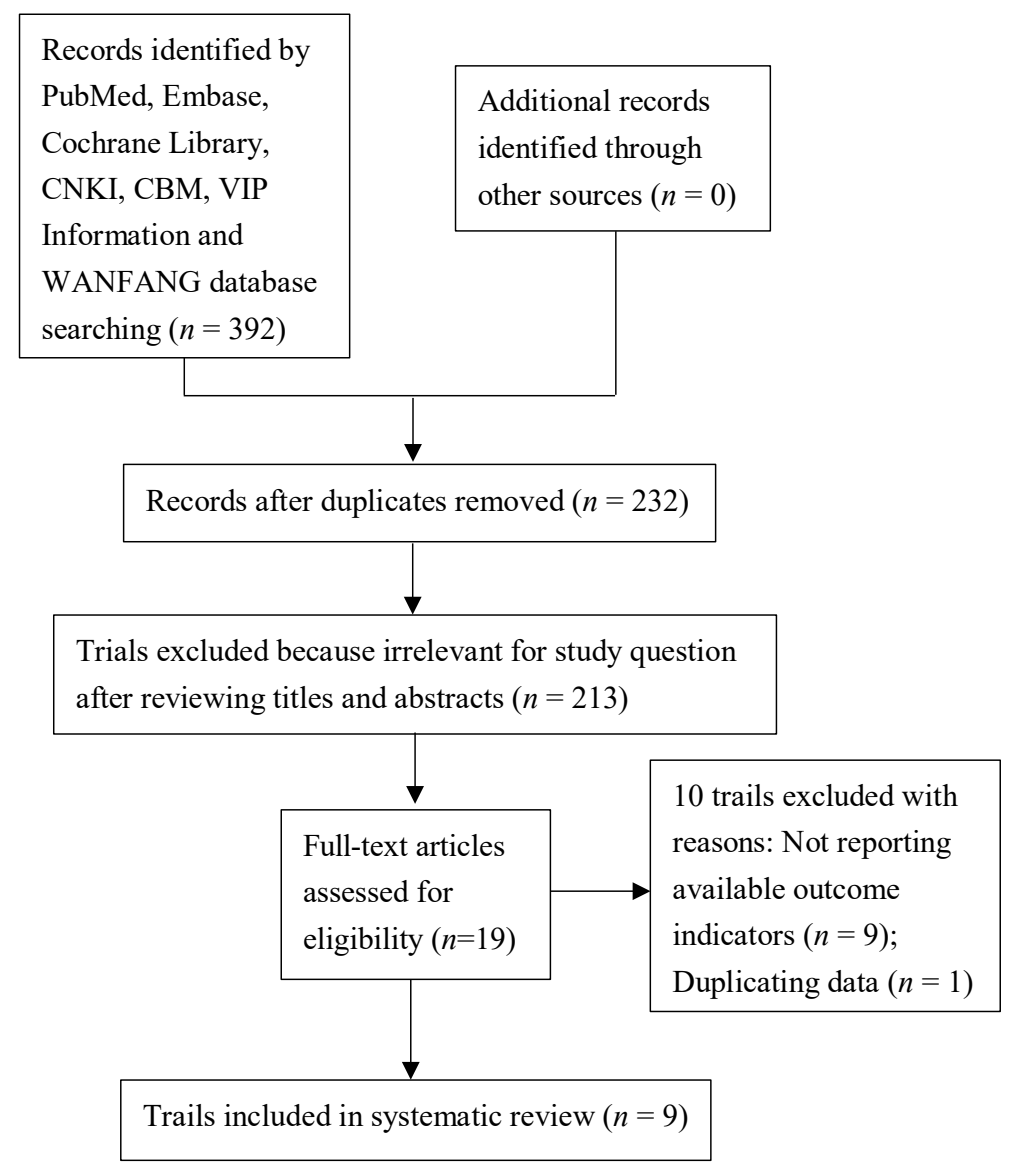

Figure 1: Flow chart of trial selection process.

the analyses were carried out using STATA 12.0 software (Stata Corp, College Station, TX, USA). A P-value $<0.05$ was considered significant for all outcomes.

\section{RESULTS}

\section{Search results and trial characteristics}

The literature search identified 392 publications; nine trials ${ }^{[8-16]}$ were included in this systematic review after screening based on the inclusion criteria. The selection process is shown in Figure 1, and the main characteristics of the included trials are provided in Table 1. All the included trials were carried out in China and reported in Chinese journals between 2012 and 2020. A total of 688 (280 males, 408 females) patients with renal anemia were identified, 344 of whom were treated with oral iron, and the others treated with SXN, the dosage of SXN was ranged from 0.5 to $1.0 \mathrm{~g}$, tid. There were three trials ${ }^{[8-10]}$ including 242 patients with renal anemia who were not dialyzed, and six trials ${ }^{[11-16]}$ including 446 patients with renal anemia who were dialyzed, with sample sizes of the individual trial varying from 40 to 134. The mean baseline hs-CRP levels in the trials ranged from $4.33 \pm 1.03$ (standard deviation) to 7.22 $\pm 2.47 \mathrm{mg} / \mathrm{L}$, and the baseline Hb levels ranged from 67.48 \pm 10.01 to $88.56 \pm 11.09 \mathrm{~g} / \mathrm{L}$. All the treatment duration was three months.

Risk-of-bias assessment of the included trials is detailed in Figure 2. Regarding sequence generation, three trials ${ }^{[1,12,14]}$ were of low risk of bias, whereas six ${ }^{[8-10,13,15,16]}$ were of unclear risk. Regarding allocation generation, six trials ${ }^{[8-10,13,15,16]}$ were of unclear risk of bias, whereas three ${ }^{[11,12,14]}$ were of high risk. All included studies ${ }^{[8-16]}$ were unblinded, which were all of high risk of bias. Six trials $s^{[8-10,12,13,16]}$ were considered as low risk for incomplete outcome data (attrition bias), and three trials ${ }^{[11,13,16]}$ were considered as high risk for selective outcome reporting (reporting bias).

\section{Hypersensitive C-reactive protein}

Nine trials ${ }^{[8-16]}$ reported on the outcome of hs-CRP. As shown in Figure 3, there was significant heterogeneity across trials $\left(I^{2}=98.4 \%, P<0.001\right)$. A random-effect model meta-analysis showed a significant beneficial effect of SXN compared with oral iron supplementation in the reduction of hs-CRP (WMD -1.93 mg/L; 95\% CI -2.14 to -1.72). Subgroup analyses (Table 2) indicated a strong effect in dialysis patients (WMD -2.15 mg/L; 95\% CI -2.37 to -1.92). 
Zeng et al: : Shengxuening versus oral iron supplementation for the treatment of renal anemia: A systematic review

\begin{tabular}{|c|c|c|c|c|c|c|c|c|c|c|c|c|}
\hline \multirow{2}{*}{$\begin{array}{l}\text { Author/ } \\
\text { year }\end{array}$} & \multirow{2}{*}{$\begin{array}{l}\text { Sample } \\
\text { sizes } \\
\text { (exp; } \\
\text { con) }\end{array}$} & \multirow[t]{2}{*}{ Age(year) } & \multirow[t]{2}{*}{$\begin{array}{l}\text { Sex } \\
\text { (M/F) }\end{array}$} & \multicolumn{2}{|c|}{$\begin{array}{l}\text { baseline hs- } \\
\text { CRP (mg/L) }\end{array}$} & \multicolumn{2}{|c|}{$\begin{array}{l}\text { Baseline } \mathrm{Hb} \\
\text { (g/L) }\end{array}$} & \multicolumn{2}{|l|}{ Intervention } & \multirow{2}{*}{$\begin{array}{l}\text { Dura- } \\
\text { tion } \\
\text { (m) }\end{array}$} & \multirow{2}{*}{$\begin{array}{l}\text { Outcome } \\
\text { measures }\end{array}$} & \multirow[t]{2}{*}{$\begin{array}{l}\text { Patient } \\
\text { type }\end{array}$} \\
\hline & & & & Exp & Con & Exp & Con & Exp & Con & & & \\
\hline $\begin{array}{l}\text { Long Li } \\
2012^{[8]}\end{array}$ & $34 ; 34$ & $\begin{array}{l}\text { exp: } 53.2 \\
\pm 12.0 \\
\text { con: } 51.6 \\
\pm 13.8\end{array}$ & $35 / 33$ & $\begin{array}{l}4.67 \\
\pm \\
2.53\end{array}$ & $\begin{array}{l}4.82 \\
\pm \\
3.15\end{array}$ & $\begin{array}{l}82.75 \\
\pm \\
10.34\end{array}$ & $\begin{array}{l}83.01 \\
\pm \\
7.98\end{array}$ & $\begin{array}{l}\text { SXN, } 0.5 \mathrm{~g} \\
\text { tid + EPO }\end{array}$ & $\begin{array}{l}\text { Oral iron } \\
\text { sulfate, } 0.3 \\
\text { g, tid + EPO }\end{array}$ & 3 & $\begin{array}{l}\text { hs-CRP, } \\
\text { Hb, Hct, } \\
\text { SF, TSAT, } \\
\text { SCR, ALB }\end{array}$ & $\begin{array}{l}\text { Non- } \\
\text { dialysis }\end{array}$ \\
\hline $\begin{array}{l}\text { Tan } \\
\text { Jinping } \\
2013^{[9]}\end{array}$ & $58 ; 58$ & $\begin{array}{l}\text { mean:52.7 } \\
\pm 11.4\end{array}$ & $55 / 61$ & $\begin{array}{l}4.67 \\
\pm \\
2.54\end{array}$ & $\begin{array}{l}4.83 \\
\pm \\
3.14\end{array}$ & $\begin{array}{l}82.74 \\
\pm 9.58\end{array}$ & $\begin{array}{l}83.14 \\
\pm \\
8.07\end{array}$ & $\begin{array}{l}\text { SXN, } 0.5 \mathrm{~g} \\
\text { tid + EPO }\end{array}$ & $\begin{array}{l}\text { Oral iron } \\
\text { sulfate, } 0.3 \\
\text { g, tid + EPO }\end{array}$ & 3 & $\begin{array}{l}\text { hs-CRP, } \\
\text { Hb, Hct, } \\
\text { SF, TSAT, } \\
\text { ALB }\end{array}$ & $\begin{array}{l}\text { Non- } \\
\text { dialysis }\end{array}$ \\
\hline $\begin{array}{l}\text { Guo Aili } \\
2015^{[10]}\end{array}$ & $29 ; 29$ & $\begin{array}{l}\text { mean:45.3 } \\
\pm 19.5\end{array}$ & $24 / 34$ & $\begin{array}{l}4.54 \\
\pm \\
2.57\end{array}$ & $\begin{array}{l}4.43 \\
\pm \\
3.47\end{array}$ & $\begin{array}{l}67.48 \\
\pm \\
10.01\end{array}$ & $\begin{array}{l}69.67 \\
\pm \\
10.56\end{array}$ & $\begin{array}{l}\mathrm{SXN}, 0.75 \mathrm{~g} \\
\text { tid }+ \text { EPO }\end{array}$ & $\begin{array}{l}\text { Oral iron } \\
\text { fumarate, } \\
0.2 \mathrm{~g} \text {, tid + } \\
\text { EPO }\end{array}$ & 3 & $\begin{array}{l}\text { hs-CRP, } \\
\text { Hb, Hct, } \\
\text { SF, TSAT, } \\
\text { SCR, } \\
\text { ALB, SI, } \\
\text { TIBC, } \\
\text { dosage of } \\
\text { EPO }\end{array}$ & $\begin{array}{l}\text { Non- } \\
\text { dialysis }\end{array}$ \\
\hline $\begin{array}{l}\text { Jin Hua } \\
2016^{[11]}\end{array}$ & $20 ; 20$ & $\begin{array}{l}\text { exp:56.24 } \\
\pm 9.6 \\
\text { con:59.5 } \\
\pm 10.6\end{array}$ & $13 / 27$ & $\begin{array}{l}7.04 \\
\pm \\
2.24\end{array}$ & $\begin{array}{l}7.22 \\
\pm \\
2.47\end{array}$ & $\begin{array}{l}87.11 \\
\pm \\
10.39\end{array}$ & $\begin{array}{l}88.56 \\
\pm \\
11.09\end{array}$ & $\begin{array}{l}\text { SXN, } 0.5 \mathrm{~g} \\
\text { tid + EPO }\end{array}$ & $\begin{array}{l}\text { Oral polysac- } \\
\text { charide iron } \\
\text { complex } \\
\text { capsule, } 150 \\
\text { mg, qd + } \\
\text { EPO }\end{array}$ & 3 & $\begin{array}{l}\text { hs-CRP, } \\
\text { IL-6, } \\
\text { Hb, Hct, } \\
\text { hepcidin, } \\
\text { SF, TSAT, } \\
\text { dosage of } \\
\text { EPO, ERI }\end{array}$ & Dialysis \\
\hline $\begin{array}{l}\text { Liu } \\
\text { Yongbing } \\
2016^{[12]}\end{array}$ & $30 ; 30$ & $\begin{array}{l}\text { mean:50.2 } \\
\pm 14.6\end{array}$ & $25 / 35$ & $\begin{array}{l}4.63 \\
\pm \\
1.46\end{array}$ & $\begin{array}{l}4.52 \\
\pm \\
1.32\end{array}$ & $\begin{array}{l}75.30 \\
\pm 5.03\end{array}$ & $\begin{array}{l}74.81 \\
\pm \\
4.83\end{array}$ & $\begin{array}{l}\text { SXN, } 1.0 \mathrm{~g} \\
\text { tid + EPO }\end{array}$ & $\begin{array}{l}\text { Oral iron } \\
\text { succinate, } \\
0.2 \mathrm{~g} \text {, tid + } \\
\text { EPO }\end{array}$ & 3 & $\begin{array}{l}\text { hs-CRP, } \\
\text { IL- } 6 \text {, } \\
\text { TNF- } \alpha \text {, } \\
\text { Hb, Hct }\end{array}$ & Dialysis \\
\hline $\begin{array}{l}\text { Zhang } \\
\text { Juan } \\
2016^{[13]}\end{array}$ & $28 ; 28$ & $\begin{array}{l}\text { mean:51.3 } \\
\pm 11.4\end{array}$ & $25 / 31$ & $\begin{array}{l}4.68 \\
\pm \\
2.38\end{array}$ & $\begin{array}{l}4.87 \\
\pm \\
2.18\end{array}$ & $\begin{array}{l}82.36 \\
\pm 9.42\end{array}$ & $\begin{array}{l}83.12 \\
\pm \\
8.78\end{array}$ & $\begin{array}{l}\text { SXN, } 0.5 \mathrm{~g} \\
\text { tid + EPO }\end{array}$ & $\begin{array}{l}\text { Oral polysac- } \\
\text { charide iron } \\
\text { complex } \\
\text { capsule, } 300 \\
\text { mg, qd + } \\
\text { EPO }\end{array}$ & 3 & $\begin{array}{l}\text { hs-CRP, } \\
\text { Hb, Hct, } \\
\text { SF, TSAT, } \\
\text { SCr, ALB }\end{array}$ & Dialysis \\
\hline $\begin{array}{l}\text { Zhou Jin } \\
2016^{[14]}\end{array}$ & $67 ; 67$ & $\begin{array}{l}\exp : 54.8 \\
\pm 3.6 \\
\text { con :55.3 } \\
\pm 4.3\end{array}$ & $55 / 79$ & $\begin{array}{l}4.85 \\
\pm \\
2.94\end{array}$ & $\begin{array}{l}4.85 \\
\pm \\
3.09\end{array}$ & $\begin{array}{l}73.89 \\
\pm 5.35\end{array}$ & $\begin{array}{l}73.92 \\
\pm \\
5.08\end{array}$ & $\begin{array}{l}\text { SXN, } 0.5 \mathrm{~g} \\
\text { tid + EPO }\end{array}$ & $\begin{array}{l}\text { Oral iron } \\
\text { dextran, } 50 \\
\text { mg, tid }+ \\
\text { EPO }\end{array}$ & 3 & $\begin{array}{l}\text { hs-CRP, } \\
\text { Hb, SF, } \\
\text { TSAT, } \\
\text { SCR, ALB }\end{array}$ & Dialysis \\
\hline $\begin{array}{l}\text { Liu Gang } \\
2018^{[15]}\end{array}$ & $40 ; 40$ & $\begin{array}{l}\exp \\
: 49.52 \pm \\
1.45 ; \\
\text { con } \\
: 50.32 \pm \\
1.08\end{array}$ & $23 / 57$ & $\begin{array}{l}4.33 \\
\pm \\
1.03\end{array}$ & $\begin{array}{l}4.63 \\
\pm \\
1.03\end{array}$ & $\begin{array}{l}82.23 \\
\pm 1.23\end{array}$ & $\begin{array}{l}83.23 \\
\pm \\
1.22\end{array}$ & $\begin{array}{l}\mathrm{SXN}, 0.5 \mathrm{~g}, \\
\text { tid }\end{array}$ & $\begin{array}{l}\text { Oral polysac- } \\
\text { charide iron } \\
\text { complex } \\
\text { capsule, } 300 \\
\text { mg, qd }\end{array}$ & 3 & $\begin{array}{l}\text { hs-CRP, } \\
\text { Hb, Hct, } \\
\text { SF, TSAT, } \\
\text { ALB }\end{array}$ & Dialysis \\
\hline $\begin{array}{l}\text { Zhong } \\
\text { Huiling } \\
2020^{[16]}\end{array}$ & $38 ; 38$ & $\begin{array}{l}\exp \\
: 50.68 \pm \\
13.28 \\
\text { con } \\
: 50.33 \pm \\
13.25\end{array}$ & $25 / 51$ & $\begin{array}{l}4.62 \\
\pm \\
1.45\end{array}$ & $\begin{array}{l}4.53 \\
\pm \\
1.32\end{array}$ & $\begin{array}{l}75.31 \\
\pm 5 . \\
03\end{array}$ & $\begin{array}{l}74.82 \\
\pm 4 . \\
84\end{array}$ & $\begin{array}{l}\mathrm{SXN} \\
0.5-1.0 \mathrm{~g} \\
\text { tid + EPO }\end{array}$ & $\begin{array}{l}\text { Oral iron } \\
\text { dextran, } 0.2 \\
\text { g, tid + EPO }\end{array}$ & 3 & $\begin{array}{l}\text { hs-CRP, } \\
\text { IL-6, } \\
\text { TNF- } \alpha, \\
\text { Hb, Hct, } \\
\text { Hcy }\end{array}$ & Dialysis \\
\hline
\end{tabular}

Exp: experimental group (SXN); Con: control group (oral iron); hs-CRP: hypersensitive C-reactive protein; Hb: hemoglobin; Hct: hematocrit value; SF: serum ferritin; TSAT: serum transferrin saturation; ALB: albumin; SCr: serum creatinine; SI: serum iron; TIBC: total iron-binding capacity; ERI: erythropoietin resistance index; IL-6: interleukin-6; TNF- $\alpha$ : tumor necrosis factor- $\alpha$; Hcy: homocysteine. 
Random sequence generation (selection bias)

Allocation concealment (selection bias)

Blinding of participants and personnel (performance bias)

Blinding of outcome assessment (detection bias)

Incomplete outcome data (attrition bias)

Selective reporting (reporting bias)

Other bias
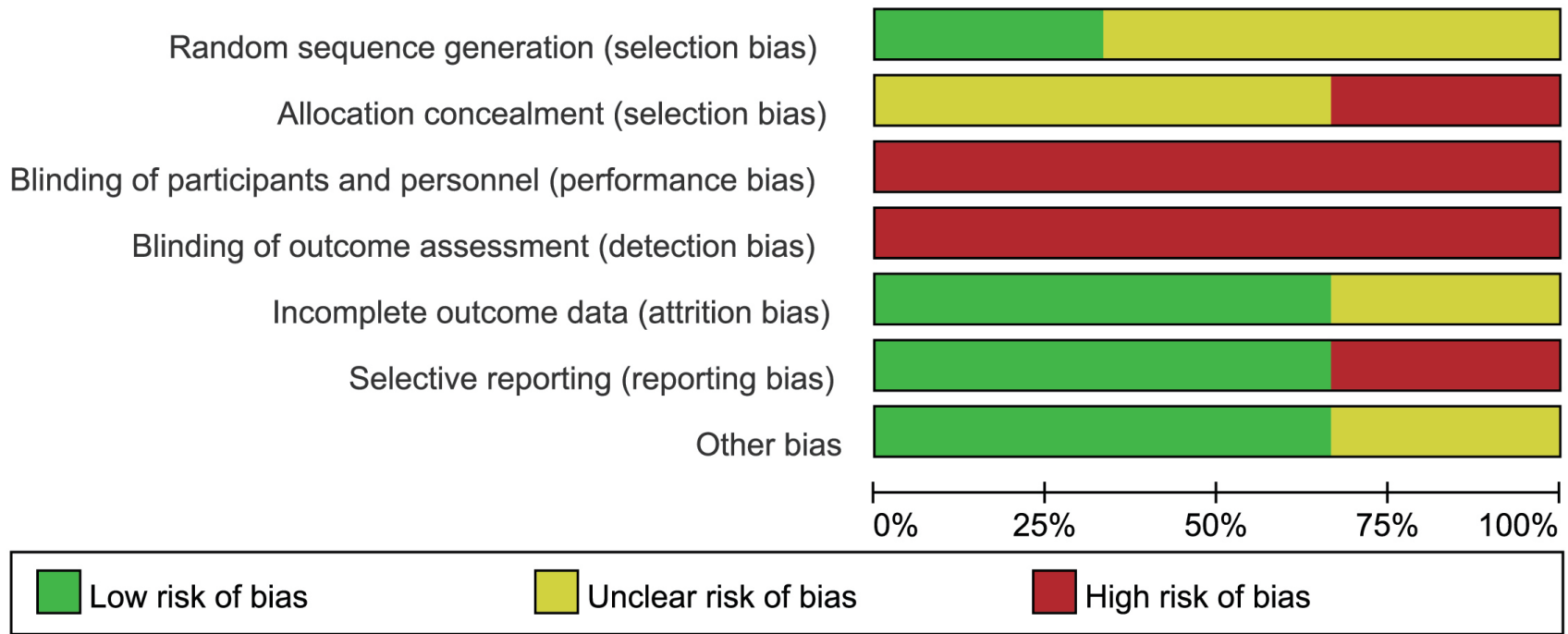

Figure 2: Risk of bias graph.

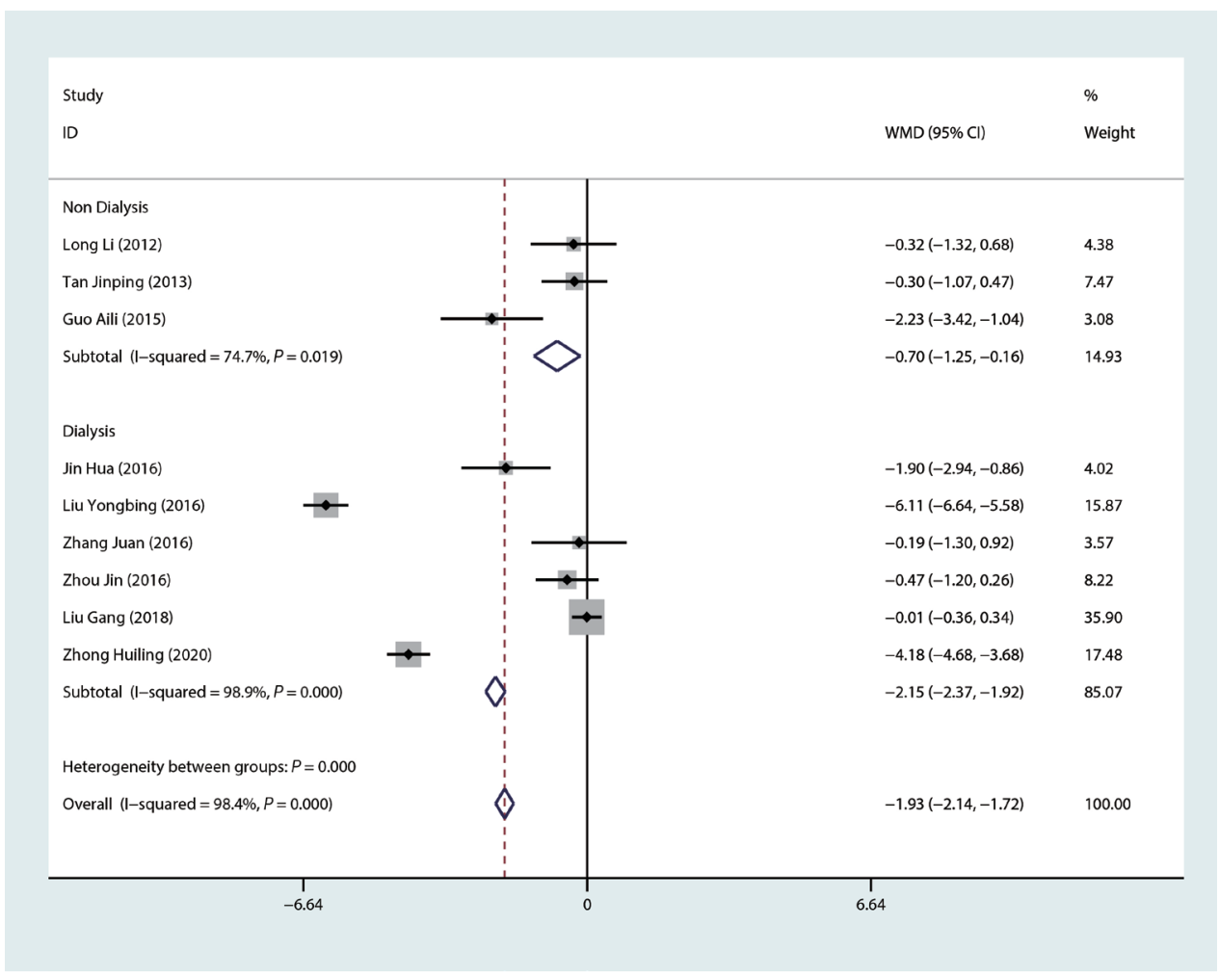

Figure 3: Forest plots of hypersensitive C-reactive protein level for SXN group vs. oral iron group.

\section{Interleukin-6 and tumor necrosis factor-a}

Two trials ${ }^{[1,12]}$ reported on the outcome of IL-6, while we did not merge the two trials' data due to the difference of data units. One trial ${ }^{[12]}$ reported on the outcome of TNF- $\alpha$. We observed that there was a significant beneficial effect of SXN compared with oral iron supplementation in the reduction of IL-6 $(P<0.05)$ and TNF- $\alpha(P<0.05)$ (date not shown).

\section{Hemoglobin}

Nine trials ${ }^{[8-16]}$ reported on the outcome of $\mathrm{Hb}$. As shown in Figure 4, there was a significant heterogeneity across trials $\left(I^{2}=97.3 \%, P<0.001\right)$. A random-effect model meta-analysis showed a significant beneficial effect of SXN compared with oral iron supplementation in increasing $\mathrm{Hb}$ (WMD $13.40 \mathrm{~g} / \mathrm{L} ; 95 \%$ CI 12.95 to 13.84). Subgroup analyses (Table 2) indicated a strong effect in 
Zeng et al.: Shengxuening versus oral iron supplementation for the treatment of renal anemia: A systematic review

\begin{tabular}{|c|c|c|c|c|}
\hline Subgroups & $\begin{array}{l}\text { Number of } \\
\text { studies }\end{array}$ & WMD & $95 \% \mathrm{Cl}$ & $\begin{array}{l}\text { Heterogeneity between } \\
\text { studies }\end{array}$ \\
\hline \multicolumn{5}{|l|}{ 1.hs-CRP } \\
\hline Non-dialysis & 3 & $-0.70 \mathrm{mg} / \mathrm{L}$ & -1.25 to -0.16 & $P=0.019 ; I^{2}=74.7 \%$ \\
\hline Dialysis & 6 & $-2.15 \mathrm{mg} / \mathrm{L}$ & -2.37 to -1.92 & $P<0.001 ; P^{2}=98.9 \%$ \\
\hline \multicolumn{5}{|l|}{ 2. $\mathrm{Hb}$} \\
\hline Non-dialysis & 3 & $15.90 \mathrm{~g} / \mathrm{L}$ & 13.86 to 117.93 & $P=0.020 ; P^{2}=74.3 \%$ \\
\hline Dialysis & 6 & $13.27 \mathrm{~g} / \mathrm{L}$ & 12.81 to 113.73 & $P<0.001 ; I^{2}=98.2 \%$ \\
\hline \multicolumn{5}{|l|}{ 3.TSAT } \\
\hline Non-dialysis & 3 & $7.42 \%$ & 6.36 to 8.49 & $P=0.072 ; l^{2}=61.9 \%$ \\
\hline Dialysis & 4 & $6.80 \%$ & 6.39 to 7.20 & $P<0.001 ; I^{2}=89.4 \%$ \\
\hline \multicolumn{5}{|l|}{ 4.SF } \\
\hline Non-dialysis & 3 & $48.87 \mu \mathrm{g} / \mathrm{L}$ & 30.66 to 67.09 & $P<0.001 ; P^{2}=97.7 \%$ \\
\hline Dialysis & 4 & $28.60 \mu \mathrm{g} / \mathrm{L}$ & 4.54 to 52.66 & $P<0.001 ; I^{2}=100 \%$ \\
\hline \multicolumn{5}{|c|}{ 5.Adverse reactions } \\
\hline Non-dialysis & 3 & 0.05 & 0.01 to 0.23 & $P=0.950 ; l^{2}=0.0 \%$ \\
\hline Dialysis & 5 & 0.14 & 0.08 to 0.52 & $P=0.480 ; l^{2}=0.0 \%$ \\
\hline
\end{tabular}

non-dialysis patients (WMD $15.90 \mathrm{~g} / \mathrm{L}$; 95\% CI 13.86 to 17.93$)$.

\section{Serum transferrin saturation}

Seven trials ${ }^{[8-11,13-15]}$ reported on the outcome of TSAT. As shown in Figure 5, there was significant heterogeneity across trials $\left(I^{2}=82.7 \%, P<0.001\right)$. A random-effect model meta-analysis showed a significant beneficial effect of SXN compared with oral iron supplementation in increasing TSAT (WMD 6.88\%; 95\% CI 6.50 to 7.26). Subgroup analyses (Table 2) indicated a strong effect in non-dialysis patients (WMD 7.42\%; 95\% CI 6.36 to 8.49).

\section{Serum ferritin}

Seven trials ${ }^{[8-11,13-15]}$ reported on the outcome of SF. As shown in Figure 6, there was significant heterogeneity across trials $\left(I^{2}=100 \%, P<0.001\right)$. A random-effect model meta-analysis showed a significant beneficial effect of SXN compared with oral iron supplementation in increasing SF (WMD $38.46 \mu \mathrm{g} / \mathrm{L} ; 95 \%$ CI 23.26 to 53.67). Subgroup analyses (Table 2) indicated a strong effect in non-dialysis patients (WMD $48.87 \mu \mathrm{g} / \mathrm{L}$; 95\% CI 30.66 to 67.09 ).

\section{Adverse reactions}

Eight trials ${ }^{[8-10,12-16]}$ reported adverse reactions. The main adverse reactions are gastrointestinal adverse reactions, including loss of appetite, bloating, nausea, constipation, and so on. As shown in Figure 7, there was no significant heterogeneity across trials $\left(I^{2}=0.0 \%, P=0.491\right)$. A fixed-effect model meta-analysis showed that oral iron supplementation was associated with an increased risk of gastrointestinal adverse reactions (RR $0.14 ; 95 \%$ CI 0.06 to 0.32) when compared with SXN. Subgroup analyses (Table 2) indicated a higher risk of gastrointestinal reactions in dialysis patients (RR 0.21 ; 95\% CI 0.08 to 0.52 ).

\section{DISCUSSION}

As nine relevant RCTs involving 688 patients with renal anemia were systematically reviewed above, we found that SXN could provide better effects on patients with renal anemia than oral iron supplementation, including: (a) reduction in the level of hs-CRP, IL-6 and TNF- $\alpha$; (b) significant rise in the level of Hb, TSAT and SF; (c) higher security with less adverse reactions.

Under normal conditions, inflammation is a protective and physiological response to various harmful stimuli. However, in several chronic debilitating disorders, such as CKD, inflammation becomes maladaptive, uncontrolled, and persistent. Systemic persistent inflammation has, for almost 20 years, been recognized as a major contributor to the uremic phenotype and a predictor of cardiovascular and total mortality. ${ }^{[17]}$ Kinetics of iron metabolism illustrate that inflammation and hepcidin can affect iron unitization. Meanwhile, inflammation and impaired renal clearance in CKD increase plasma hepcidin, which will inhibit duodenal iron absorption and sequestering iron in macrophages. These effects of hepcidin can cause systemic iron deficiency, decreased availability of iron for erythropoiesis, and resistance to endogenous and exogenous EPO. ${ }^{[18]}$ Importantly, inflammation may cause hyporesponsiveness to ESA, which is associated with poor prognosis in patients with renal anemia. ${ }^{[19]}$ Generally speaking, inflammation is more severe in dialysis patients than non-dialysis patients. Therefore, it is a crucial challenge to be settled urgently that how to reduce the level of inflammation and hepcidin for patients with renal anemia.

Patients with renal anemia who have an absolute or functional iron deficiency were all recommended to treat with 
Study

ID
$\%$

WMD $(95 \% \mathrm{Cl})$

Weight

Non Dialysis

Long Li (2012)

Tan Jinping (2013)

Guo Aili (2015)

Subtotal (I-squared $=74.3 \%, P=0.020$ )

Dialysis

Jin Hua (2016)

Liu Yongbing (2016)

Zhang Juan (2016)

Zhou Jin (2016)

Liu Gang (2018)

Zhong Huiling (2020)

Subtotal (I-squared $=98.2 \%, P=0.000)$

Heterogeneity between groups: $P=0.014$

Overall (I-squared $=97.3 \%, P=0.000$ )
$17.24(13.53,20.95) \quad 1.46$

$16.85(14.16,19.54) \quad 2.78$

$8.19(2.40,13.98) \quad 0.60$

$15.90(13.86,17.93) \quad 4.83$

$18.01(11.58,24.44) \quad 0.49$

$-2.69(-6.21,0.83) \quad 1.62$

$14.13(10.36,17.90) \quad 1.41$

$16.94(15.25,18.63) \quad 6.99$

$14.00(13.50,14.50) \quad 80.46$

$-1.49(-3.68,0.70) \quad 4.21$

$13.27(12.81,13.73)$

$13.40(12.95,13.84) \quad 100.00$

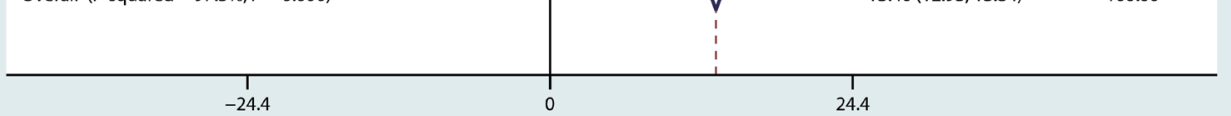

Figure 4: Forest plots of hemoglobin level for SXN group vs. oral iron group.

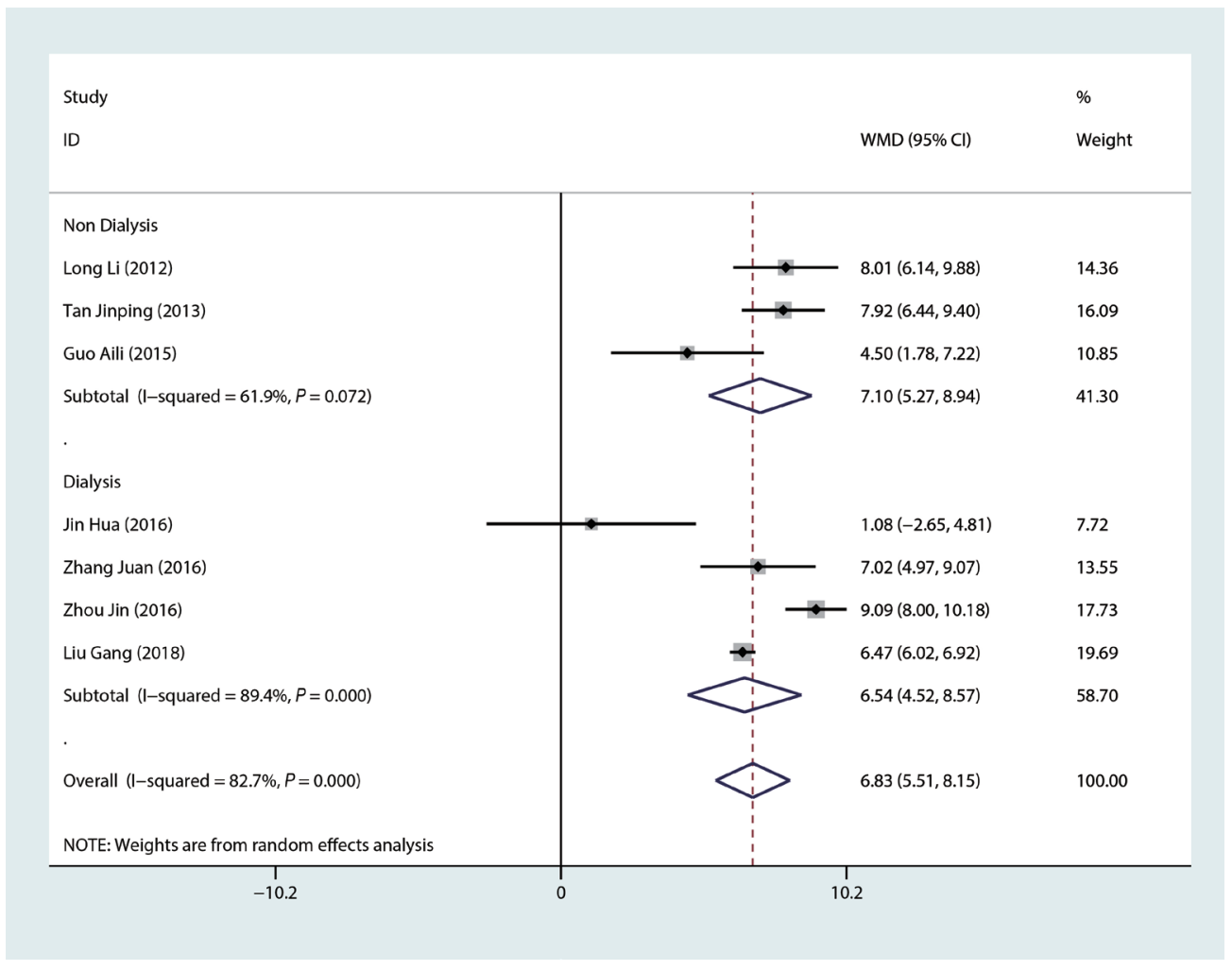

Figure 5: Forest plots of serum transferrin saturation level for SXN group vs. oral iron group. 


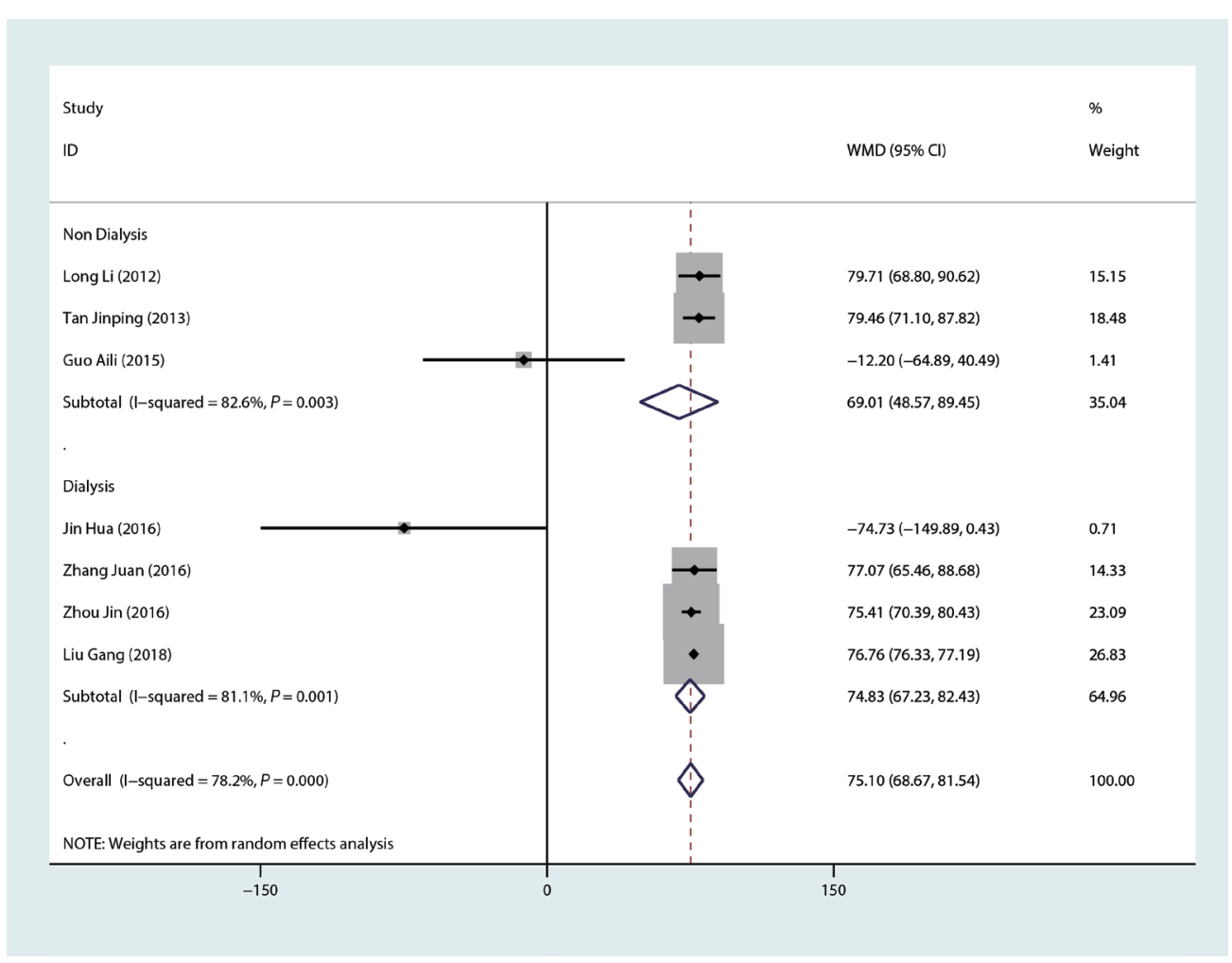

Figure 6: Forest plots of serum ferritin level for SXN group vs. oral iron group.

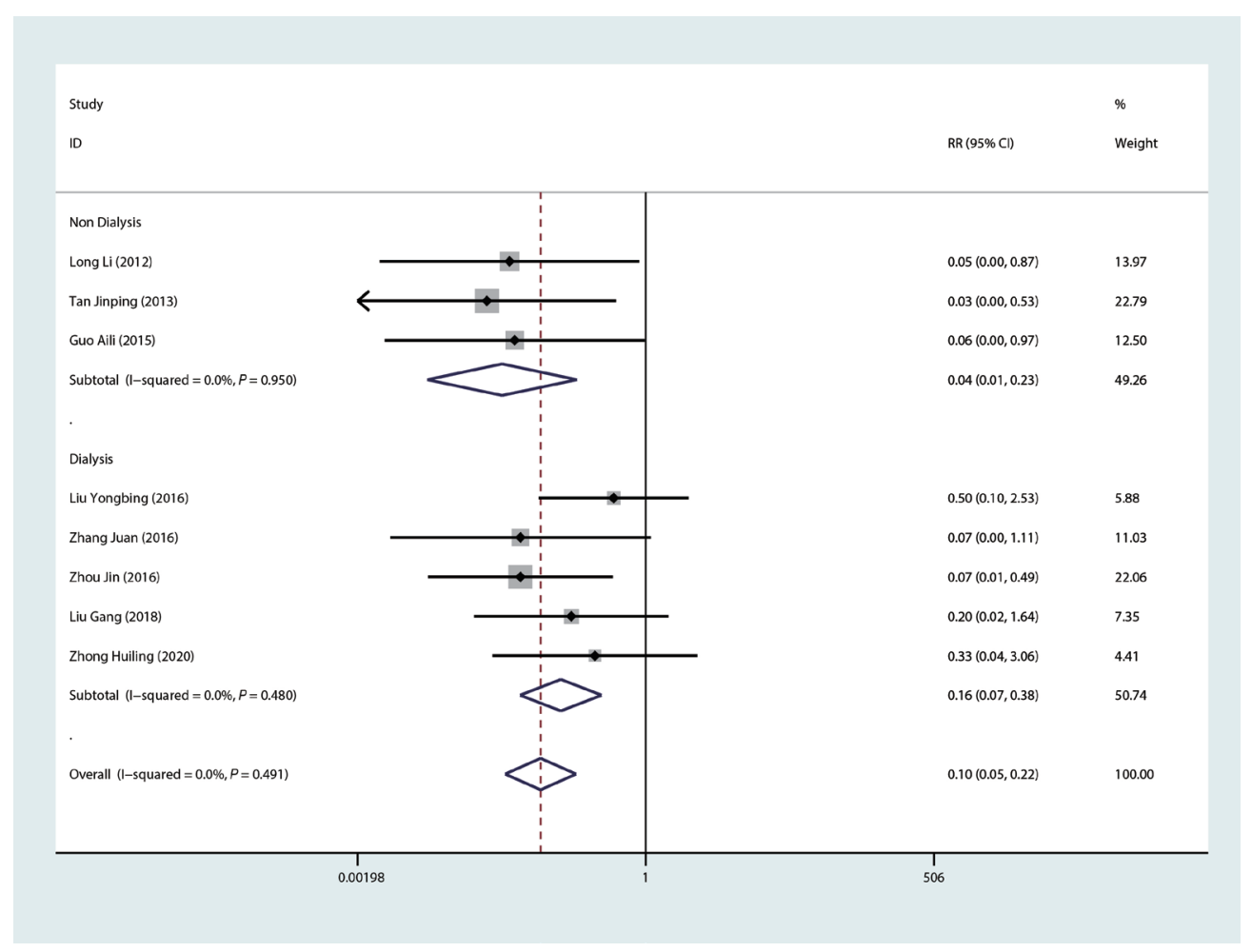

Figure 7: Forest plots of adverse reaction for SXN group vs. oral iron group. 
iron supplementation. ${ }^{[4]}$ However, it may cause iron overload (serum ferritin $\geq 500 \mu \mathrm{g} / \mathrm{L}$ ) for patients with functional iron deficiency. Serum ferritin is the main form of iron storage in the body, which can reflect the iron reserve. Furthermore, many studies have found elevated levels of hs-CRP in patients taking oral iron, ${ }^{[8-10,12-16]}$ which may indicate that oral iron supplementation has a risk of increasing inflammation. And oral iron is mainly absorbed in the duodenum and upper jejunum mucosa with an acid environment ${ }^{[20,21]}$ which probably causes gastrointestinal reaction.

A study demonstrated that silkworm feces extract (SFE) treatment improved hematological parameters, decreased total iron-binding capacity, increased serum iron, transferrin saturation, liver iron and spleen iron of iron deficiency anemia rats. Western blot analysis showed that SFE can upregulate iron-regulatory protein (IRP) expression and downregulate ferritin expression in livers. Furthermore, SFE can inhibit hepcidin expression and increase ferroportin1 expression by blocking JAK2/ STAT3, BMP6/SMAD, and HFE/TfR2 pathways. And, SFE also can attenuate hepcidin expression induced by IL-6 and BMP6 in HepG2 cells. ${ }^{[2]}$ The main component of SXN is SFE, which means SXN may improve anemia and iron metabolism disorder via reducing the level of inflammation and hepcidin. Furthermore, the animal experiment also showed that SXN had similar effects with EPO ${ }^{[23]}$ which could obviously promote the proliferation of erythroid and granulocyte cells of bone marrow in normal mice and increase the proportion of peripheral blood reticulocyte. It has suggested that $\mathrm{SXN}$ can increase the synergistic effect with EPO and decrease the dosage of EPO, which were consistent with clinical findings from Guo $\mathrm{AL}^{[10]}$ and Jin H. ${ }^{[1]}$ We observed that the level of serum ferritin, which was closed to or already in the state of iron overload, had reduced after treated with SXN in the trail of Jin $\mathrm{H},{ }^{[1]]}$ while other included trails ${ }^{[8-10,12-16]}$ had opposite results, so we consider that SXN can reduce the level of serum ferritin when iron overload and promote it when absolute iron deficiency.

Chemical analysis and structural verification showed that SXN belongs to a ferrous derivative, which was mainly composed of Fe chlorin p6, Fe chlorin e6, and Fe isochlorin e4. ${ }^{[24]}$ Chlorophyll contained in SXN has the same structure with human heme, so it can directly compound hemoglobin, which increases the bioavailability. ${ }^{[25]}$ The organic hematin chloride in SXN is similar to human hematoporphyrin, which can be immediately absorbed by the intestinal mucosal cells with less irritation. ${ }^{[2]}$ And a series of studies showed that SXN has the lowest incidence of adverse reactions compared with ferrous sulfate, ferrous fumarate, polysaccharide iron complex capsule, ferrous succinate, and ferrous gluconate. ${ }^{[27-29]}$
Several limitations in our systematic review should be noted. First, a publication bias was inevitable because all included studies were from the same country. Second, the sample size was small, especially the number of non-dialysis patients was small. Third, the quality of included studies was poor, only three trails reported the specific randomized method and the others only mentioned "random". All included trails were unblinded. Some secondary endpoints were not reported in several studies, this could potentially cause reporting bias. Forth, significant statistical heterogeneity existed, even though we have done subgroups analysis. The possible explanation for it may be correlated with small sample size and poor quality of included studies. Finally, EPO can effectively raise the level of hemoglobin, while only two trails recorded the dosage of EPO, so we were not able to verdict accurately whether the difference of increasing hemoglobin between the two groups was associated with different doses of EPO, which would increase the limitations of our results.

\section{CONCLUSION}

According to this systematic review, SXN is more effective and safer than oral iron supplementation in the treatment of renal anemia, especially in reducing the level of inflammation. In the future, high-quality, large sample size, and multicenter trials are necessary to confirm these findings, which must record the dosage of EPO and then evaluate the effect of SXN on EPO dosage systematically.

\section{Author contributions and declarations}

Zeng Q wrote the article as the first author and worked with Wang XH on the topic selection, literature selection, data extraction, and system analysis of the article. Yang LP was responsible for handling the different opinions between Zeng Q and Wang XH. Lang R and Liang Y polished the article and $\mathrm{Yu} \mathrm{RH}$ has been coaching in this process as the correspondence author. This manuscript has been read and approved by all of the authors for publication.

\section{Conflict of Interest}

There is no conflict of interest existed in the submission of this manuscript.

\section{REFERENCES}

1. Regidor DL, Kopple JD, Kovesdy CP, Kilpatrick RD, McAllister CJ, Aronovitz J, et al. Associations between changes in hemoglobin and administered erythropoiesis-stimulating agent and survival in hemodialysis patients. J Am Soc Nephrol 2006;17:1181-91.

2. Babitt JL, Lin HY. Mechanisms of anemia in CKD. J Am Soc Nephrol 2012;23:1631-4. 
3. Icardi A, Paoletti E, De Nicola L, Mazzaferro S, Russo R, Cozzolino M. Renal anemia and EPO hyporesponsiveness associated with vitamin D deficiency: the potential role of inflammation. Nephrol Dial Transplant 2013;28: 1672-9.

4. Kidney Disease: Improving Global Outcomes (KDIGO) Anemia Work Group. KDIGO clinical practice guideline for anemia in chronic kidney disease. Kidney Int Suppl 2012; 2:279-335.

5. Miret S, Tascioglu S, van der Burg M, Frenken L, Klaffke W. In vitro bioavailability of iron from the heme analogue sodium iron chlorophyllin. J Agric Food Chem 2010;58:1327-32.

6. Zhang L, Zhang W, Jin H, Wang D, Wei N, Wang Y. Effect and safety of Shengxuening (extract from excrement of bombyxin) for renal anemia: a systematic review. J Tradit Chin Med 2016;36:588-95.

7. Higgins JPT, Green S, eds. Cochrane Handbook for Systematic Reviews of Interventions. Version 5.1.0 [updated March 2011]. The Cochrane Collaboration 2011. Available from: http://www.cochrane-handbook. org/. Access on June 1, 2020.

8. Long L, Wu YL, Lu YH, Ally. [Clinical observation of shengxuening tablet in the treatment of renal anemia in chronic kidney disease patients]. Changchun Zhongyiyao Daxue Xuebao = J Changchun Univ Tradit Chin Med 2012; 28: 504-6.

9. Tan JP, Wen SJ, Guan RY, Liu GZ, Chen FX. [Clinical analysis of Shengxuening in the treatment of 58 cases of Renal Anemia]. Zhongguo Yiliao Qianyan $=$ China Healthcare Innovation 2013;8:37-8.

10. Guo AL. [Clinical observation of Shengxuening in the treatment of renal anemia in non-dialysis patients with chronic kidney disease]. Linchuang Shenzangbing Zazhi = J Clin Nephrol 2015;15:285-8.

11. Jin $\mathrm{H}$, Kong $\mathrm{M}$, Wang $\mathrm{YP}$, Cheng W. [Effect of Sericularia extract on Erythropoietin Resistance in hemodialysis patients based on the mechanism of iron metabolism regulated by iron modulators]. Linchuang Shenzangbing Zazhi = J Clin Nephrol 2016;16:294-8.

12. Liu YB, Hu DJ. [Clinical observation of Shengxuening tablet in reducing the risk of microinflammation in maintenance hemodialysis patients with renal anemia]. Zhongguo Yaofang = China Pharm 2016;27:1096-8.

13. Zhang J, Wu XY. Clinical observation of [Shengxuening tablet in treatment of renal anemia in patients with maintenance peritoneal dialysis]. Linchuang Shenzangbing Zazhi = J Clin Nephrol 2016;16:492-5.

14. Zhou J, Guo FL, Dong QR, Huang L. [Effect of Shengxuening tablet on renal anemia in patients undergoing diabetic nephropathy peritoneal dialysis]. Zhongguo Laonianxue Zazhi = Chin J Gerontol 2016;36:5383-4.

15. Liu G. Clinical effect of [Shengxuening tablet on renal anemia in patients with maintenance peritoneal dialysis]. Linchuang Yiyao Wenxian Zazhi = J Clin Med Liter 2018;5:94-5.126.
16. Zhong HL, Wu YL, Liao XY. Observation on the effect of [Shengxuening tablet in reducing the risk of microinflammatory reaction in maintenance hemodialysis patients with renal anemia]. Jilin Yixue $=$ Jilin Med J 2020;41:116-8.

17. Cobo G, Lindholm B, Stenvinkel P. Chronic inflammation in end-stage renal disease and dialysis. Nephrol Dial Transplant 2018;33(Suppl 3): iii35-40.

18. Ganz T, Nemeth E. Iron balance and the role of hepcidin in chronic kidney disease. Semin Nephrol 2016;36:87-93.

19. Nangaku M. Pathogenesis and treatment of anemia in chronic kidney disease. Rinsho Ketsueki 2017; 58: 1860-3.

20. Conrad ME, Umbreit JN. Iron absorption and transport-an update. Am J Hematol 2000;64: 287-98.

21. Fidler MC, Davidsson L, Zeder C, Hurrell RF. Erythorbic acid is a potent enhancer of nonheme iron absorption. Am J Clin Nutr 2004;79: 99-102.

22. Huang X, Wu J, Li Q, Gu SS, Chen ZH, Wang KP. Silkworm feces extract improves iron deficiency anemia via suppressing hepcidin expression and promoting iron-regulatory proteins expression. RSC Advances 2017; 7: 50378-88.

23. Liu XL, Chen K, Shi H, Cai HF, Li LM, Lou JY, et al. Effects of sodium iron chlorophyllin on colony formation of progenitor cells and experimental anemia in normal mice (in Chinese). Chin J Hematol 1997;18:234-6.

24. Nie J, Hu HS, Chen XY, Liu JJ, Li K, Luo J. [Study on the fingerprint of Shengxuening tablets.] Zhongguo Zhongyao Zazhi = Chin J Chin Materia Med 2013; 20: 3502-6.

25. Carpenter CE, Mahoney AW. Contributions of heme and nonheme iron to human nutrition. Crit Rev Food Sci Nutr 1992; 31: 333-67.

26. Shayeghi M, Latunde-Dada GO, Oakhill JS, Laftah AH, Takeuchi K, Halliday $\mathrm{N}$, et al. Identification of an intestinal heme transporter. Cell 2005; 122: 789-801.

27. Cancelo-Hidalgo MJ, Castelo-Branco C, Palacios S, Haya-Palazuelos J, Ciria-Recasens M, Manasanch J. et al. Tolerability of different oral iron supplements: a systematic review. Curr Med Res Opin 2013;29:291-303.

28. Klein-Schwartz W. Toxicity of polysaccharide-iron complex exposures reported to poison control centers. Ann Pharm 2000;34:165-9.

29. Ding L, Xu L, Jin Y, Wei Y, Pan Y, Sattar S, et al. Efficacy of SXN in the treatment of iron deficiency anemia: A phase IV clinical trial. Evid Based Complement Alternat Med 2019; ID8796234.

How to cite this article: Zeng $Q$, Wang $X H$, Yang LP, Lang $R$, Liang $Y$, $\mathrm{Yu} \mathrm{RH}$. Shengxuening versus oral iron supplementation for the treatment of renal anemia: A systematic review. J Transl Intern Med 2020; 8: 245-54. 